\title{
STUDIES OF CHAOTIC BEHAVIOUR IN HERA CAUSED BY TRANSVERSE MAGNETIC MULTIPOLE FIELDS
}

\author{
H. Meis, A. Wrulich \\ Deutsches Elektronen-Synchrotron DESY, 2000 Hamburg 52, W.-Germany
}

F. Schmidt

II. Physikalisches Institut der Universität Hamburg

\section{Abstract}

The relevance of chaotic behaviour to the HERA proton ring is studied. Special emphasis is placed on finding and investigating stochastic trajectories in phase space. The concept of characteristic Lyapunov exponents is applied to various model systems such as two- and four-dimensional maps

\section{Introduction and formulation of the problem}

The Hamiltonian describing the transverse particle motion in a storage ring is given by:

$H=\frac{P^{2}}{2}+K_{x}(s) \frac{x^{2}}{2}+\frac{P^{2}}{2}+K_{z}(s) \frac{z^{2}}{2}+\sum_{n, m} a_{n m}(s) x^{n} z^{m}$

with

$x, z \quad \ldots$ transverse displacements of the particle with respect to a fixed reference orbit

$P_{x}, P_{z} \ldots$ conjugate momenta

$K_{x}, K_{z} \ldots$ focusing strengths in the horizontal and vertical plane

a (s).. multipole expansion coefficients of the applied fields

s ....... arclength along the reference trajectory

The nonlinearities are characterized by the coefficients $a(s)$ and they are either introduced artificially e.nm. by sextupoles which compensate the natural chromaticity or they occur naturally as deviations from linear fields due to errors. If, as in the HERA proton ring, superconducting magnets are used then these field errors are the dominant contribution to the nonlinearity.

The equations ofmotion derived from (1) are highly nonlinear and in general constitute a non-integrable system. The general theory of these systems / / predicts regular KAM trajectories and chaotic trajectories in phase space characterized by an extremely sensitive dependence on initial conditions. A quantitative measure of stochasticity (chaos) is given by the characteristic Lyapunov exponent. Roughly speaking, this exponent describes how the distance between two adjacent points in phase space evolves with time. The rormal definition is

$$
x=\lim _{t \rightarrow \infty} \frac{1}{t} \ln \left|\frac{d(t)}{d(0)}\right|
$$

with $d(t)$ phase space distance at time $t$

$d(o)$ initial phase space distance.

Regular trajectories show a linear increase of $d(t)$ whereas stochastic trajectories separate exponentially.

In order to investigate the influence of nonlinear fields quantitatively, the fast computer code RACETRACK has been developed $/ 2 /$, which allows one to take into account nonlinearities up to 20 -poles in the thin lens approximation. In this approximation the solution to Hamilton's equations of motion is given in form of nonlinear four-dimensional maps.
One way to use tracking codes is to look at the maximum stable initial amplitude of a particle in phase space i.e. we look at those trajectories which have the maximum initial amplitude for which the particle motion remains bounded or finite up to the number of revolutions which it is practical to track These maximum amplitudes define the four-dimensional dynamical aperture.

In the following sections we present numerical results using RACETRACK with special emphasis on finding and investigating chaotic trajectories in phase space.

The calculations have been performed on a $370 \mathrm{E}$ Emulator and the IBM $3081 \mathrm{~K}$. The number of revolutions was varied between 30000 and 300000 using a HERA optics with a fixed realistic multipole distribution due to norlinear field errors in the superconducting magnets.

\section{Numerical results}

The separation of adjacent phase space points as a method of diagnosing chaos has already been used in accelrator physics in investigations of the beam- beam interaction /3/ and as a first step we have used this method for investigating pure horizontal motion (i.e. without coupling to the vertical betatron motion). For regular motion with oscillation amplitudes well within the dynamical aperture the trajectory separation increases linearly as expected but chaos was found close th the limit of the dynamical aperture. Fig. I shows a section of such a trajectory with a highly enlarged scale.

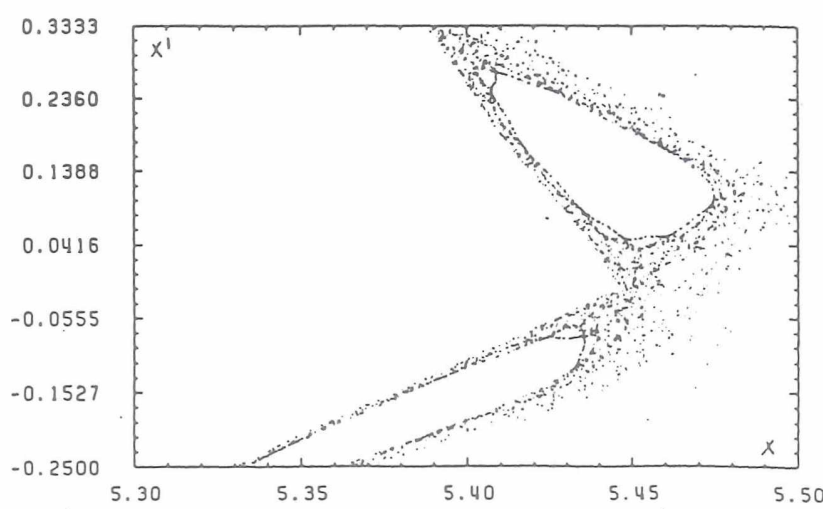

Fig. 1: Chaotic motion of a particle close to the dynamical aperture

The exponential growth of the distance between trajectories starting at two adjacent points in this chaotic region is shown in Fig. 2. The saturation is due to the boundedness of the available phase space. 


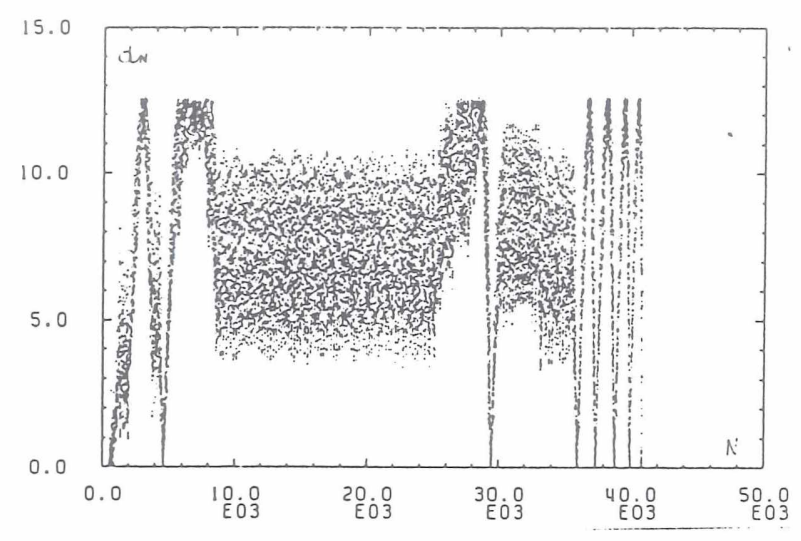

Fig. 2: Separation of trajectories for chaotic motion

Whereas these results follow nicely the general scenario of chaos in nonlinear two-dimensional maps as described for example in $/ 1 /$, little is known about the onset of stochasticity in four- and higher-dimensional maps. The calculations performed for the fully coupled betatron oscillations are just a first step in this direction. The results are summarized in Fig.'s 3 to 8 . Fig's 3 and 4 show the projections of a regular orbit onto the $x-p$-plane and the $x-z-p l a n e$ respectively. The separation of trajectories is linear as expected and is demonstrated in Fig. 5.

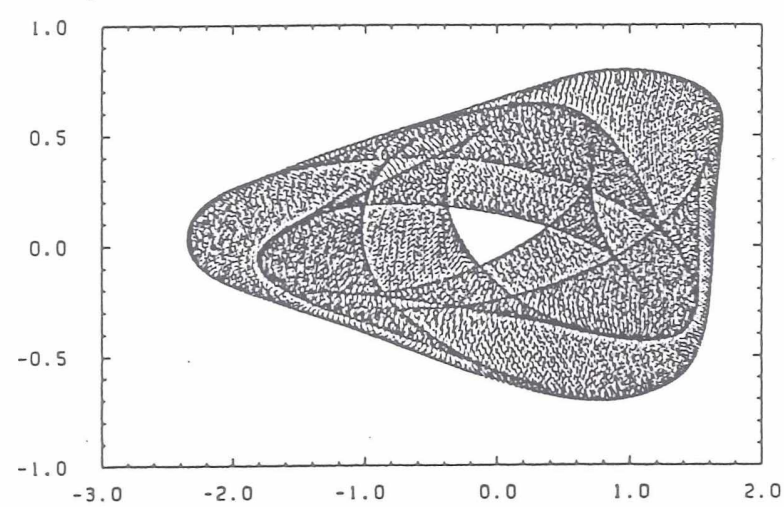

Fig. 3: Regular motion: projection onto $x-p_{x}-p l a n e$

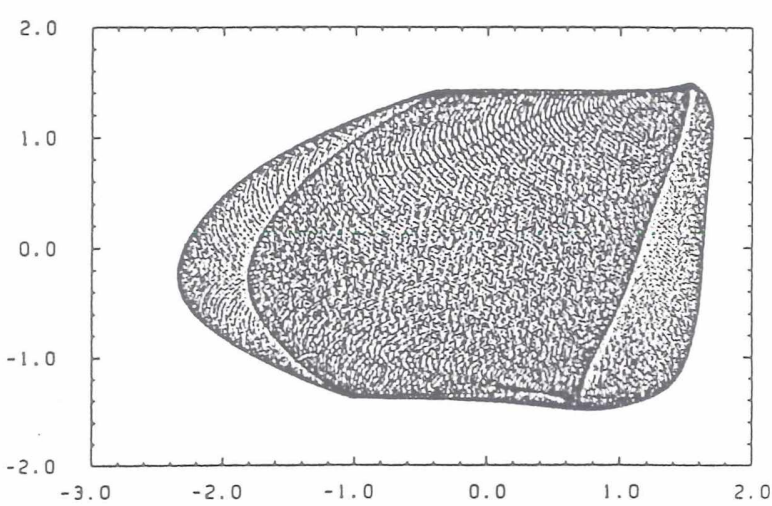

Fig. 4: Regular motion: projection onto $x-z-p l a n e$

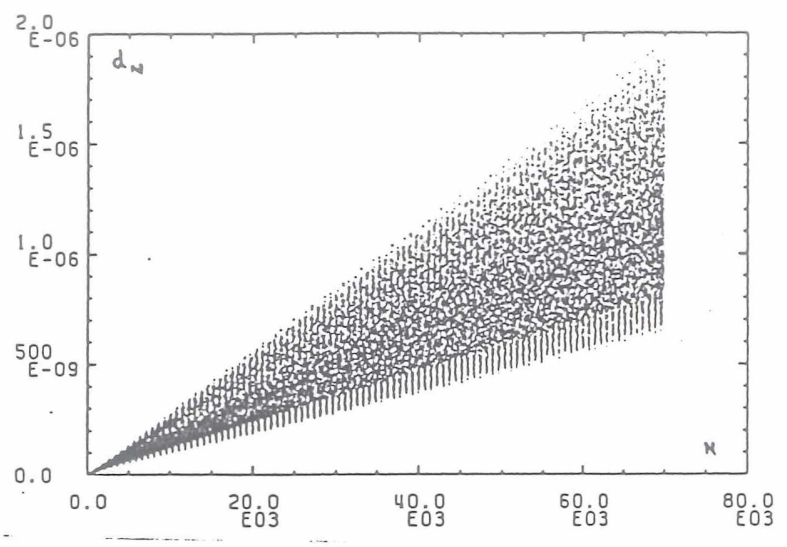

Fig. 5: Separation of regular trajectories

Fig.'s 6,7 and 8 are the corresponding pictures of typical stochastic trajectories which again can be observed close to the dynamical aperture and which do not lie on smooth two dimensional surfaces.

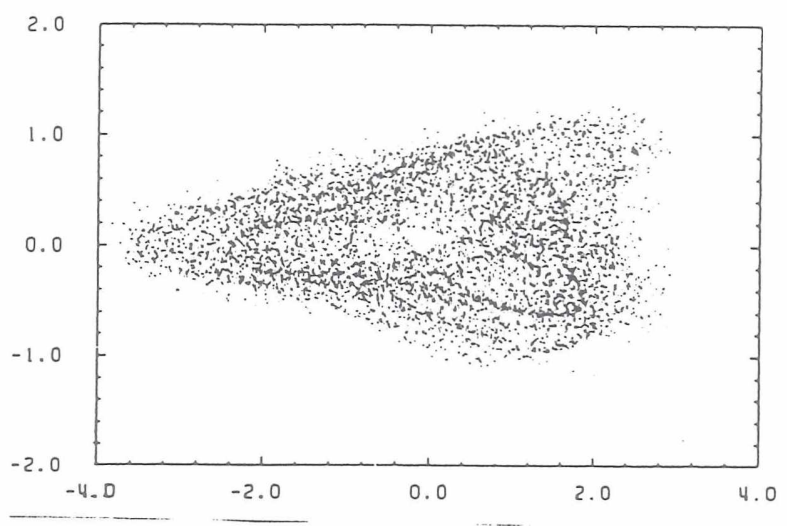

Fig. 6: Chaotic motion: projection onto $x-p_{x}-p l a n e$

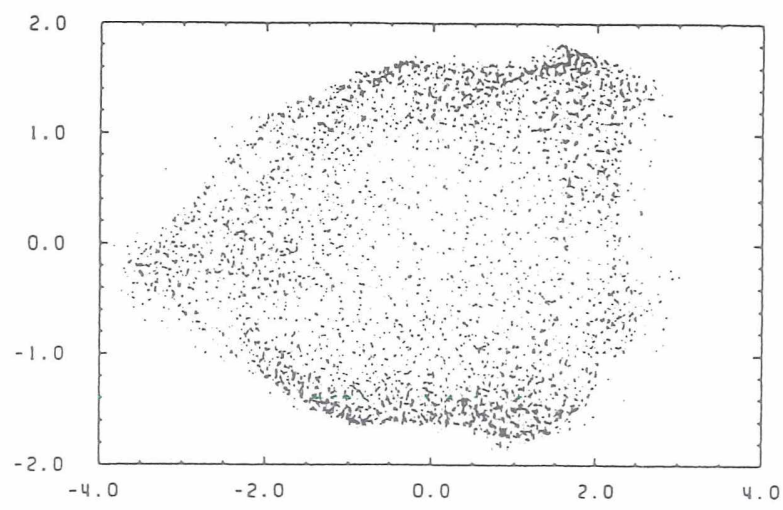

Fig. 7: Chaotic motion: projection onto $x-z$ plane 


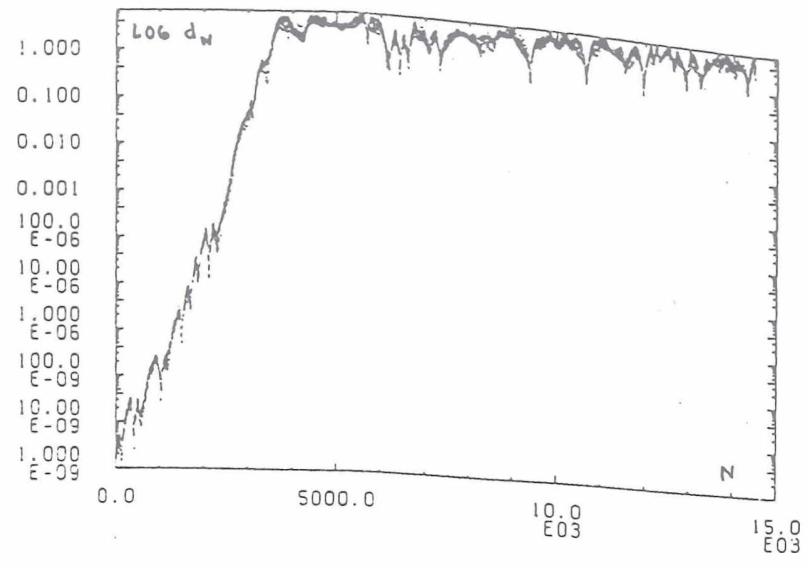

Fig. 8: Separation of chaotic trajectories (logarithmic plot)

\section{Summary and conclusions}

Numerical calculations for transverse particle motion have been performed for a HERA proton ring with nonlinear multipole fields. Until now, detectable chaotic regions have only been observed near the limit of the dynamical aperture both in the uncoupled case and in the coupled case. The calculations for the uncoupled two-dimensional case show all the features normally associated with chaotic behaviour in nonlinear two-dimensional maps. In this case the existence of KAM trajectories also implies stability of the system. However, because of the possibility of Arnold diffusion / $/$ / such a stability criterion cannot be derived from the existence of invariant KAM surfaces in the four-dimensional case. Also, relatively little is known about the break up of these two-dimensional surfaces in four-dimensional phase space and further investigations are certainly needed for a better understanding of the pictures 5 to 8 . The situation is even more complicated since a complete picture of the single particle dynamics in storage rings with nonlinear fields must also include the effect of synchrotron oscillations thus leading to the study of six-dimensional nonlinear maps.

\section{Acknowledgements}

The authors want to thank Dr. D. P. Barber for careful reading of the manuscript.

\section{References}

/1/ see for example

A.J. Lichtenberg, M. A. Lieberman "Regular and stochastic motion" Springer Verlag, Berlin (1983)

/2/ A. Wrulich, DESY 84-026 (1984)

/3/ D. Neuffer, A. Riddiford, A. G. Ruggiero "Searches for Arnold diffusion and chaotic motion in the beam-beam interaction" Proceedings of the Particle Accelerator Conf., Santa Fe, 1983 